\title{
Improving the Sensitivity of Chipless RFID Sensors: The Case of a Low-Humidity Sensor
}

\author{
Giada Marchi ${ }^{1,2}\left(\right.$, Viviana Mulloni ${ }^{1} @$, Omar Hammad Ali ${ }^{1}\left(\right.$, Leandro Lorenzelli ${ }^{1}$ and Massimo Donelli ${ }^{3, *}(\mathbb{1})$ \\ 1 Centre for Sensors and Devices, Fondazione Bruno Kessler, 38123 Trento, Italy; gmarchi@fbk.eu (G.M.); \\ mulloni@fbk.eu (V.M.); ohammadali@fbk.eu (O.H.A.); lorenzel@fbk.eu (L.L.) \\ 2 Department of Information Engineering and Computer Science, University of Trento, 38123 Trento, Italy \\ 3 Department of Civil Environmental and Mechanical Engineering, University of Trento, 38123 Trento, Italy \\ * Correspondence: massimo.donelli@unitn.it
}

Citation: Marchi, G.; Mulloni, V.; Hammad Ali, O.; Lorenzelli, L.; Donelli, M. Improving the Sensitivity of Chipless RFID Sensors: The Case of a Low-Humidity Sensor. Electronics 2021, 10, 2861. https://doi.org/ 10.3390 /electronics10222861

Academic Editor: Giovanni Andrea Casula

Received: 27 October 2021

Accepted: 18 November 2021

Published: 20 November 2021

Publisher's Note: MDPI stays neutral with regard to jurisdictional claims in published maps and institutional affiliations.

Copyright: (c) 2021 by the authors. Licensee MDPI, Basel, Switzerland. This article is an open access article distributed under the terms and conditions of the Creative Commons Attribution (CC BY) license (https:/ / creativecommons.org/licenses/by/ $4.0 /)$.

\begin{abstract}
This study is supposed to introduce a valid strategy for increasing the sensitivity of chipless radio frequency identification (RFID) encoders. The idea is to properly select the dielectric substrate in order to enhance the contribution of the sensitive layer and to maximize the frequency shift of the resonance peak. The specific case of a chipless sensor suitable for the detection of humidity in low-humidity regimes will be investigated both with numerical and experimental tests.
\end{abstract}

Keywords: chipless RFID sensors; microwave systems; humidity sensor; sensing materials; Nafion 117; sensor sensitivity

\section{Introduction}

In the last years, chipless radio frequency identification solutions have been proposed as a frontier technology between two leaders of the sector: the chip-based RFID and the optical barcode. The ambition of chipless RFIDs is to merge in a unique technology the advantages brought by chipped RFIDs and by barcodes, overcoming thus their less functional characteristics. For this reason, chipless RFID systems are generally composed of a passive tag and a readout architecture that exploit non-line-of-sight wireless principles to communicate. This is for sure the greatest advantage that chipless RFIDs have when compared to barcodes. At the same time, the prices of barcodes are attractive for the market if compared with those of chipped RFID tags. Chipped RFIDs indeed require application-specific integrated circuit (ASIC) chips to store the identification information, which results in preventing their use in industrial applications where the cost of tags is comparable with the cost of goods. For this reason chipless RFID investigators aim at finding a solution in this sense and simplify the tag manufacture process encoding the identification information directly in the physical structure of the tag without the use of silicon chips. The fabrication process is thus made simpler and compatible with low-cost mass production printing strategies [1,2]. Moreover, the passive tag can be impressed directly on the product surface becoming a remotely readable non-invasive label.

However, as with all good stories, this technology will reach a more mature stage but is yet under development with some clear open challenges. A typical chipless RFID tag is composed of conductive resonators on a dielectric substrate and the information is encoded in the resonance peaks that they generate in the time or frequency domain. The absence of a silicon chip affects their performance in terms of coding capability and reading distance [3]. The coding capability is strongly connected to the number of resonance structures the tag is able to physically host. In this sense, the research trend is looking at miniaturizing the resonance geometries in order to increase the number of coding structures in the tag without losing signal quality $[4,5]$. At the same time, chipless tags rely only on backscattering mechanisms to activate the tag and impress the ID signature in the response signal, and this is not the optimal way to reach long reading distances. Therefore, another 
current trend is searching for mechanisms to increase the remote interrogation capability since looking at them as completely passive labels is attractive for particular application fields, such as harsh environments [6,7]. Finally, the last trend is trying to investigate the potential use of chipless RFID as smart labels by combining their identification feature with sensing properties $[8,9]$. This last field is the particular area of interest to the current work.

Focusing on frequency-domain encoders, they can be turned into sensors by adding a suitable layer of sensing material covering the metallic resonance structures. This layer interacts with a specific physical parameter or chemical substance present in the environment and modifies accordingly the amplitude and the frequency of the encoding resonance peak. Despite the great opportunities introduced by the first prototypes reported in the literature for RFID chipless sensors, they are at the very first stages and much effort must be spent in this direction yet. The first problem is that the target is generally to detect and monitor changes due to a unique parameter. However, sensitive materials are usually affected by the presence of water particles in the air, which distorts the results and leads to an incorrect interpretation of the final data $[10,11]$. Therefore, the first focus in the chipless sensor area should be to finitely characterize chipless humidity probes, which should guide in quantifying the real impact of other chipless sensing signatures. The starting point for efficient sensor design is to screen for a well-performing sensing material or mechanism. Studies on chipless sensors for relative humidity $(\mathrm{RH})$ detection have widely investigated the performance of sensitive materials such as polyvinyl-alcohol (PVA), Kapton or even common paper considering, in this case, the substrate as the sensing component [12-15]. In this work, the sensitive material we considered is a polymer well-known by experts of the fuel cells area, but it is probably the first time it is investigated in the chipless humidity sensing context. This material is Nafion 117 and one of our previous studies [16] has already produced some satisfactory results demonstrating Nafion 117 is a very good candidate for humidity sensing. Indeed, from Nafion 117 studies [12,17] it is outlined that it has a great affinity to water and is far more sensitive than Kapton, which has a lower dielectric properties variation with respect Nafion 117, while if compared to PVA and common paper Nafion 117 shows better mechanical properties and works in an absolutely reversible way.

What we are proposing here will be a general validation of a Nafion 117-based chipless sensor targeted for low-humidity conditions. Low-humidity sensors are essential in several applications such as storage of medicine or pharmaceutical products, low moisture foods (LMF), or general products stored in an inert environment where moisture infiltration is critical. In particular, we will demonstrate that in this humidity range a substrate characterized by a low-dielectric constant is expected to perform better, in terms of sensitivity, than high-dielectric constant substrates. The study exploits the sensor proposed as an example in order to present an opportunity for discussion on the mutual impact the substrate and the sensing material have on the final sensitivity performance of chipless sensors. What is supported is that the contribution in the frequency signature of the sensitive material can be maximized by properly choosing the substrate based on the dielectric properties of the sensitive material while sensing. The idea supported can then become a useful and valid principle in the design phase of sensing material-based chipless encoders. The paper is organized as follows: in Section 2 the theoretical explanation supporting the choice of the substrate is presented along with some simulation data, in Section 3 the characterization of a low-humidity chipless sensor is provided with some experimental tests, and finally, in Section 4 some last conclusions are drawn.

\section{Materials and Methods}

The main component of the encoding structure is a microstrip resonator which generates narrow resonance peaks in the microwave frequency regime when excited by a suitable electromagnetic wave. The performance of these conductive resonators are strongly dependent on two quantities, the resonant geometry design with particular interest to its design length and the dielectric permittivity of the RF substrate. Moreover, in the case of chipless sensors, also the presence of a sensitive layer impacts the nominal behavior and must be 
considered in the design phase. Generally speaking, for a resonator gap-coupled with a $50 \Omega$-microstrip transmission line as in Figure 1 all these influences can be enclosed in the following formula taken from [18]:

$$
f_{r e s} \approx \frac{1}{\sqrt{\varepsilon_{e f f}}} \frac{c}{2 L_{r e s}}
$$

where $f_{\text {res }}$ is the first resonance frequency generated by the resonant geometry, $c$ is the light velocity, $L_{r e s}$ is the resonator length and $\varepsilon_{e f f}$ is the effective dielectric constant that in our case is a variable of prime importance. Indeed, the effective dielectric constant includes the effects due to the dielectric substrate and those due to the sensitive layer.

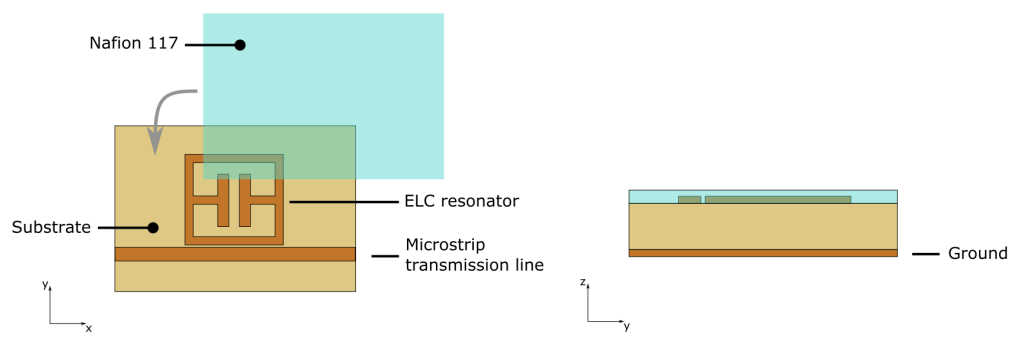

Figure 1. Design scheme of the considered ELC resonator, gap-coupled with a microstrip transmission line and covered by Nafion 117.

Here we assume of having already selected a well-performing resonant geometry for sensing purposes (in our case an electric-field-coupled (ELC) resonator according to [17]) and we try to go a step further. As seen in [16] and re-proposed in Table 1, when RH levels are low Nafion 117 tends to be characterized by medium dielectric constant $\varepsilon_{r}$ and low loss $\operatorname{tangent} \tan \delta$ values. When instead Nafion 117 starts to incorporate more water both of them increase due to the dielectric properties of water, but the effects due to the change of loss tangent value dominate. Moreover, by the electromagnetic simulations and experimental tests of study reported in [16], it is possible to understand that for high-humidity levels the resonance peak is much shifted in frequency due to higher dielectric constant values, but it appears particularly broadened due to the dominant high loss tangent values. On the other hand, for low-humidity levels, the sensor response is dominated by the variation of $\varepsilon_{r}$ of Nafion 117, which causes the frequency shift while the peak remains extremely sharp thanks to the low loss tangent values.

Table 1. Dielectric properties of Nafion 117 as in [16] used for modeling low RH states.

\begin{tabular}{lccc}
\hline Material & RH Correspondance [19] & $\varepsilon_{r}$ & $\tan \delta$ \\
\hline Nafion117 $\lambda=1$ & $\approx 0.3 \%$ & 4 & 0.0125 \\
Nafion117 $\lambda=2$ & $\approx 3 \%$ & 5 & 0.02 \\
Nafion117 $\lambda=3$ & $\approx 33 \%$ & 7 & 0.5714 \\
\hline
\end{tabular}

Here we will focus on this last case, proposing a strategy to increase the sensitivity of chipless sensors in low-humidity regimes. Only low RH values are considered in order to work in a condition of sharp resonance peaks. Our idea is that a substrate with low $\varepsilon_{r}$ can increase the sensitivity of the whole configuration compared to a substrate with high $\varepsilon_{r}$. The physical explanation for this behavior can be found in the microstrip transmission line theory, precisely in the way the electric field propagates and diffuses into the dielectric surrounding the microstrip line and the resonator (Figure 2) [20]. As already mentioned, $\varepsilon_{e f f}$ is a combination of the values of the sensing material and the substrate. When the substrate has a high $\varepsilon_{r}$, the electric field lines are more bent towards the substrate and, consequently, the contribution of the substrate to the effective dielectric constant is high 
and the contribution of the sensing material is low. In this case, the sensitivity in terms of frequency shift of the sensor results to be drastically reduced. Conversely, using a substrate with a low $\varepsilon_{r}$, the contribution of the sensing material to the effective dielectric constant is more important, and the frequency shift and sensitivity higher.

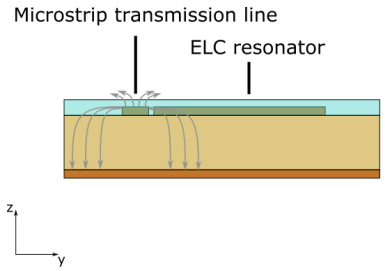

(a) $\varepsilon_{\mathrm{r}_{\text {sub }}}>\varepsilon_{\mathrm{r}_{\text {sensitive }}}$

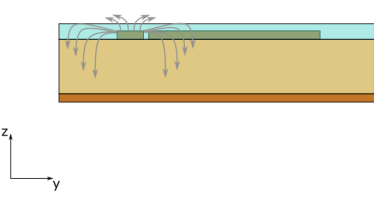

(b) $\varepsilon_{\mathrm{r}_{\text {sub }}}<\varepsilon_{\text {rsensitive }}$

Figure 2. E-field line scheme in the case (a) the substrate has $\varepsilon_{r_{\text {sub }}}>\varepsilon_{r_{\text {sensitive }}}$ which results in a major impact of the substrate in the final frequency signature of the resonator and (b) the substrate has $\varepsilon_{r_{\text {sub }}}<\varepsilon_{r_{\text {sensitive }}}$ which results in a major impact of the sensitive layer in the final frequency signature of the resonator.

In order to support our paper, we performed a set of simulations with the Ansys High Frequency Structure Simulator (HFSS). The ELC resonator design is the same reported in [16], and a comparison among a higher dielectric constant substrate Rogers $\mathrm{RO} 4003$ $\left(\varepsilon_{r}=3.28, \tan \delta=0.0027 @ 10 \mathrm{GHz}\right.$, thickness of $\left.h=0.8 \mathrm{~mm}\right)$ used in [16] and a lower dielectric constant substrate DiClad $\left(\varepsilon_{r}=2.33, \tan \delta=0.0013 @ 10 \mathrm{GHz}\right.$, thickness of $h=0.8$ $\mathrm{mm}$ ) is proposed for low-humidity levels $(<35 \%)$. The substrates have been chosen since they are comparable in terms of thickness and loss tangent value in order to understand the impact of their relative permittivity. Since according to Equation (1) a change of substrate (and therefore of $\varepsilon_{r}$ ) would generate a resonance peak of the ELC resonator at a different frequency, we have properly scaled the resonator dimensions on the DiClad substrate match the resonance peak frequency of simulations in [16]. Its final size on DiClad are 13.4 $\times 13.4 \mathrm{~mm}^{2}$. After this calibration process, a $150 \mu \mathrm{m}$ thick-Nafion 117 layer was added to the configuration. The dielectric parameters of Nafion 117 (Table 1) used as in [16] are related to different $\mathrm{RH}$ conditions measured at $25^{\circ} \mathrm{C}$. In the table the variable $\lambda$ indicates the average number of water molecules surrounding the sulfonic group in the polymeric structure of Nafion 117 and a rough correspondence with the RH level is also proposed from [19].

The results of the simulation process for DiClad are compared in Figure 3 with those of Rogers RO4003 of [16]. From the plot it is possible to appreciate the initial calibration process of the ELC resonator on the two substrates, which has been performed in order to start having them resonating at the same resonance frequency of $2.8 \mathrm{GHz}$ without Nafion 117. Even though the difference in the $\varepsilon_{r}$ of the two substrates is not very large, the different frequency shift of the resonance peak is well visible from the simulation. A more pronounced frequency shift is generated by the lowest relative permittivity substrate DiClad, which results in a higher sensitivity sensor. 


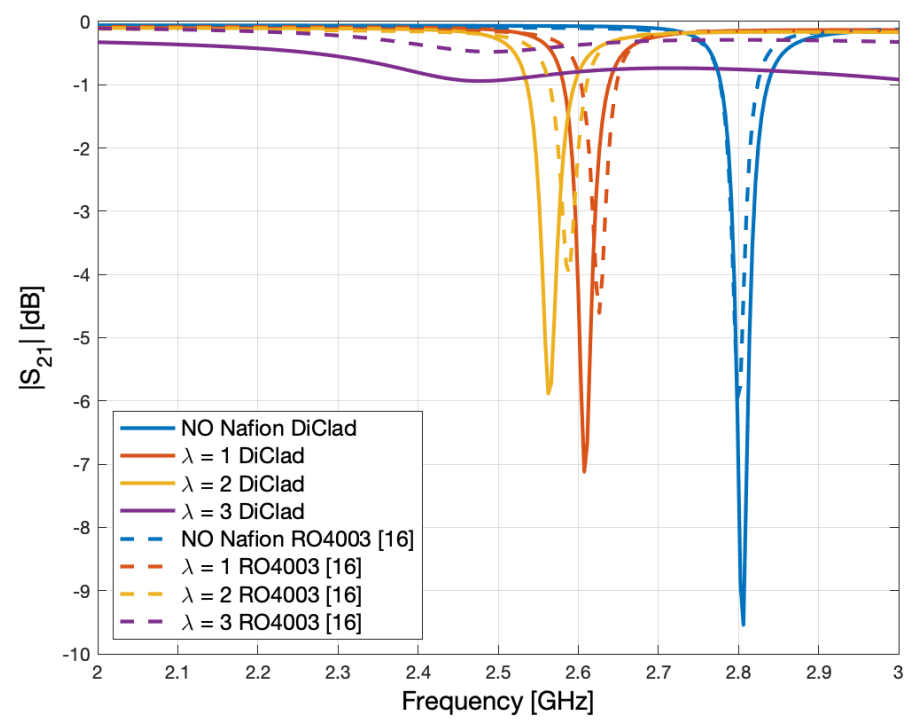

Figure 3. Evaluation of the substrate impact on the sensitivity of a humidity chipless sensor in low-humidity conditions. Simulation of a $2.8 \mathrm{GHz}$ ELC resonator covered by Nafion 117 on DiClad $\left(\varepsilon_{r}=2.33\right)$ and Rogers RO4003 $\left(\varepsilon_{r}=3.28\right)$ [16] laminates.

\section{Results}

Since from the previous section, simulations supported the idea that for low or very low RH measurements, using a substrate with an $\varepsilon_{r}$ as low as possible is the best choice, the sensor on DiClad has been fabricated and tested in order to propose a better candidate for low-humidity measurements. The sensing configuration has been fabricated by $\mathrm{CNC}$ milling, equipped with Sub-Miniaturize type A (SMA) connectors and placed in a climatic chamber as in [16]. Its S-parameters have been properly monitored by means of a vector network analyzer (VNA) connected via RF cables to the sensor. The climatic chamber has been set up to work at a fixed temperature $\left(25^{\circ} \mathrm{C}\right)$ and humidity up to $45 \%$. Figure 4 plots the variation of the first resonance peak for $\mathrm{RH}$ up to $25 \%$. We decided to report only the acquisitions up to $\mathrm{RH} 25 \%$ since the sensor is designed to work well at low-humidity but is less performing at higher humidity due to the broadening of the resonance peak. The discrepancies in terms of the frequency range of the peaks that can be noticed comparing simulation and measurement results are known and well explained in [16]. They are due to the dielectric values of Nafion 117 used in the simulation process which were not specifically studied for the use case of Nafion 117 in chipless sensors. Nevertheless, they result to be a good guiding line to verify the electromagnetic behavior of Nafion 117 as a sensitive layer for chipless sensors. A more precise correspondence could be found by reversely characterizing its dielectric values from chipless sensitive tests. 


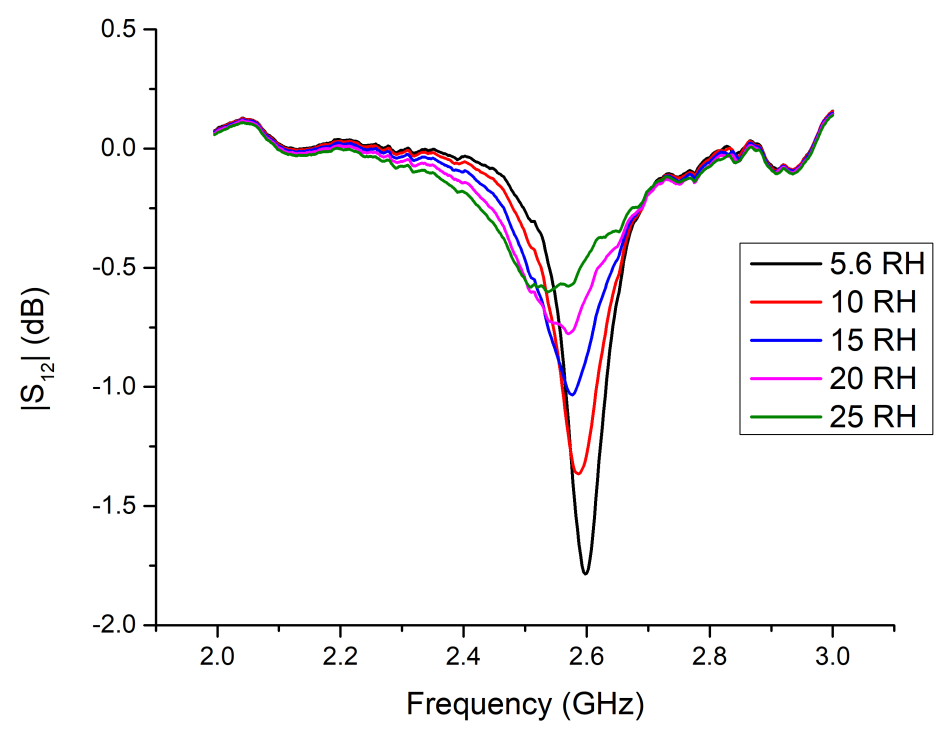

Figure 4. Insertion loss measurements at varying relative humidity of a Nafion 117 based humidity sensor on DiClad substrate. The measurements have been performed in climatic chamber for lowhumidity regimes.

Figure 5 shows that as humidity increases the sensor prototype presents a linear shift of the resonance peak toward lower frequencies, while the variation of the peak intensity is not linear. As a consequence, it could be strategic to monitor its frequency shift in order to take advantage of this linearity.

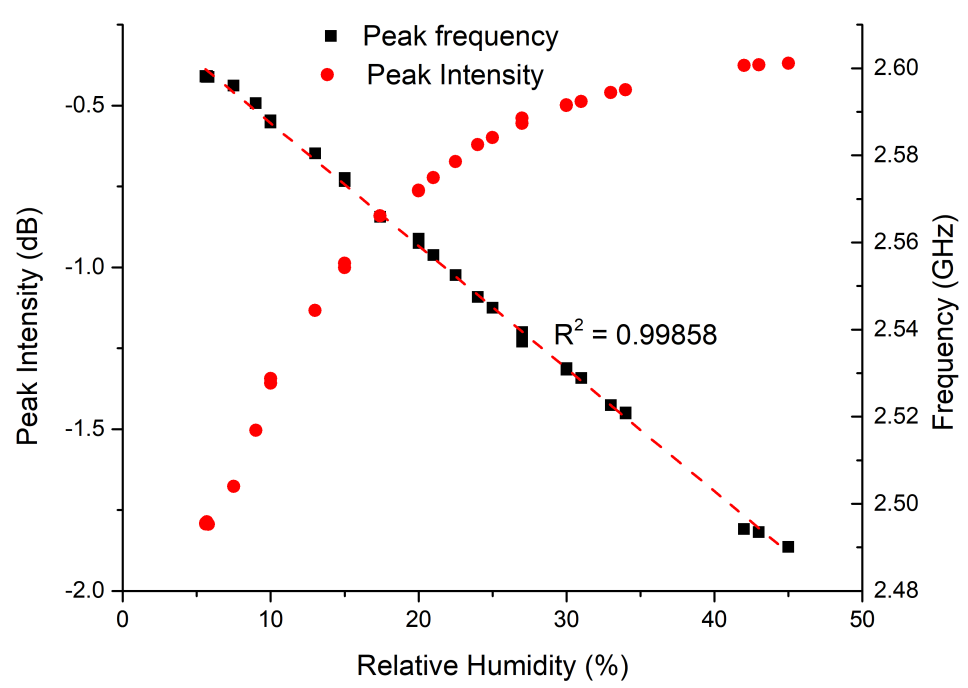

Figure 5. Frequency shift and peak intensity variation for varying relative humidity in the range $5-45 \%$ at $25^{\circ} \mathrm{C}$, where $\mathrm{R}$ is the Pearson correlation coefficient.

Considering the frequency shift, we can quantify the sensitivity improvement obtained within the sensor prototype on DiClad if compared with that on $\mathrm{RO} 4003$ proposed in [16]. We will consider for this comparison data related to RH 10\% and 20\% since both studies include these humidity values. We discover that our theory is experimentally well supported and verified since the sensor on $\mathrm{RO} 4003$ has a sensitivity of $11.2 \mathrm{MHz} / \mathrm{RH} \%$, while the sensor on DiClad is characterized by a more than doubled sensitivity of 27.17 
$\mathrm{MHz} / \mathrm{RH} \%$. In general, thus, given the nature of chipless tags, it is recommended to look for mechanisms to enhance their discrimination capability. With this work we have demonstrated that in the case of chipless sensors exploiting as detection mechanism the linear frequency shift the sensitivity can be appropriately tailored by choosing the most suitable substrate for the selected sensitive layer.

Finally, in Figure 6 we presented some results of the experiments made to test the sensor in its repeatability and ability of real-time sensing properties. We repeated some $10-30 \%$ RH transactions. The sensor proves to follow well the climatic chamber behavior and the sensor response and recovery times are only limited by the climatic chamber transaction times.

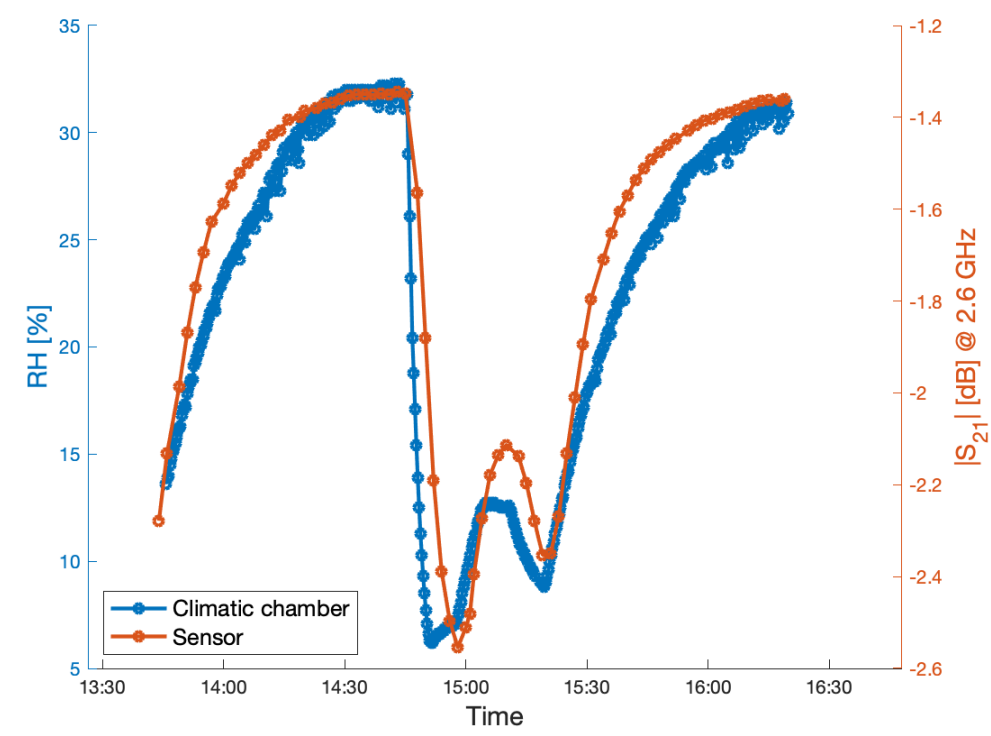

Figure 6. Sensor response times monitored at a $2.6 \mathrm{GHz}$ single frequency for some $10-30 \% \mathrm{RH}$ climatic chamber transactions.

\section{Conclusions}

Humidity is an important environmental parameter that is often interfering with chipless RFID sensor performance, and needs therefore to be precisely quantified. In this paper, a chipless RFID sensor suitable for specific RH detection in low-humidity conditions was proposed. The sensor on a DiClad dielectric substrate and with a Nafion 117 sensitive layer has been firstly electromagnetically simulated and then its performance tested in a climatic chamber at a fixed temperature of $25^{\circ} \mathrm{C}$ and humidity $<45 \% \mathrm{RH}$. We demonstrated to have doubled its sensitivity with respect to the case on RO4003 substrate [16]. Moreover, both the concepts that the low dielectric constant substrates are the best solution in the lower humidity range and the substrate has a great impact in increasing the sensitivity of chipless RFID sensors with frequency shift encoding are explained in detail. The results proposed are only a first demonstration of the principle supported and are specific for the low-humidity case. Future work will investigate many different substrates in terms of $\varepsilon_{r}$, and other optimized solutions for the high-humidity range will be analogously proposed.

Author Contributions: Conceptualization, G.M., V.M. and M.D.; methodology, G.M., V.M. and M.D.; validation, G.M. and V.M.; formal analysis, G.M. and V.M.; investigation, G.M., V.M. and O.H.A.; resources, O.H.A., M.D. and L.L.; data curation, G.M. and V.M.; writing-original draft preparation, G.M.; writing-review and editing, V.M., M.D. and L.L.; visualization, G.M., V.M., M.D. and L.L.; supervision, V.M., M.D. and L.L. All authors have read and agreed to the published version of the manuscript.

Funding: This research received no external funding.

Conflicts of Interest: The authors declare no conflict of interest. 


\section{References}

1. Khan, S.; Lorenzelli, L.; Dahiya, R.S. Technologies for Printing Sensors and Electronics Over Large Flexible Substrates: A Review. IEEE Sens. J. 2015, 15, 3164-3185. [CrossRef]

2. Purushothama, J.M.; Lopez-Soriano, S.; Vena, A.; Sorli, B.; Susanti, I.; Perret, E. Electronically Rewritable Chipless RFID Tags Fabricated Through Thermal Transfer Printing on Flexible PET Substrates. IEEE Trans. Antennas Propag. 2021, 69, $1908-1921$. [CrossRef]

3. Herrojo, C.; Paredes, F.; Mata-Contreras, J.; Martín, F. Chipless-RFID: A Review and Recent Developments. Sensors 2019, 19 , 3385. [CrossRef] [PubMed]

4. Ghiri, R.E.; Entesari, K. A 50.7-Bit Retransmission-Based Chipless RFID Tag with Miniaturized Resonators. In Proceedings of the 2021 IEEE Topical Conference on Wireless Sensors and Sensor Networks (WiSNeT), San Diego, CA, USA, 17-20 January 2021; pp. 12-14. [CrossRef]

5. Jalil, M.E.B.; Rahim, M.K.A.; Mohamed, H.; Samsuri, N.A.B.; Murad, N.A.; Dewan, R.; Majid, H.B.A.; Nafis, N.B.M.; Nur, L.O.; Nugroho, B.S. High Capacity and Miniaturized Flexible Chipless RFID Tag Using Modified Complementary Split Ring Resonator. IEEE Access 2021, 9, 33929-33943. [CrossRef]

6. Deif, S.; Daneshmand, M. Multiresonant Chipless RFID Array System for Coating Defect Detection and Corrosion Prediction. IEEE Trans. Ind. Electron. 2020, 67, 8868-8877. [CrossRef]

7. Henry, D.; Hester, J.G.D.; Aubert, H.; Pons, P.; Tentzeris, M.M. Long-Range Wireless Interrogation of Passive Humidity Sensors Using Van-Atta Cross-Polarization Effect and Different Beam Scanning Techniques. IEEE Trans. Microw. Theory Tech. 2017, 65, 5345-5354. [CrossRef]

8. Dey, S.; Saha, J.K.; Karmakar, N.C. Smart Sensing: Chipless RFID Solutions for the Internet of Everything. IEEE Microw. Mag. 2015, 16, 26-39. [CrossRef]

9. Mulloni, V.; Donelli, M. Chipless RFID Sensors for the Internet of Things: Challenges and Opportunities. Sensors 2020, $20,2135$. [CrossRef] [PubMed]

10. Athauda, T.; Karmakar, N.C. The Realization of Chipless RFID Resonator for Multiple Physical Parameter Sensing. IEEE Internet Things J. 2019, 6, 5387-5396. [CrossRef]

11. Gopalakrishnan, S.; Waimin, J.; Raghunathan, N.; Bagchi, S.; Shakouri, A.; Rahimi, R. Battery-Less Wireless Chipless Sensor Tag for Subsoil Moisture Monitoring. IEEE Sens. J. 2021, 21, 6071-6082. [CrossRef]

12. Amin, M.; Karmakar, N.; Winther-Jensen, B. Polyvinyl-alcohol (PVA)-based RF humidity Sensor in microwave frequency. Prog. Electromagn. Res. B 2013, 54, 149-166. [CrossRef]

13. Amin, E.M.; Bhuiyan, M.S.; Karmakar, N.C.; Winther-Jensen, B. Development of a Low Cost Printable Chipless RFID Humidity Sensor. IEEE Sens. J. 2014, 14, 140-149. [CrossRef]

14. Amin, E.M.; Karmakar, N.C. Development of a low cost printable humidity sensor for chipless RFID technology. In Proceedings of the 2012 IEEE International Conference on RFID-Technologies and Applications (RFID-TA), Nice, France, 5-7 November 2012; pp. $165-170$.

15. Feng, Y.; Xie, L.; Chen, Q.; Zheng, L.R. Low-Cost Printed Chipless RFID Humidity Sensor Tag for Intelligent Packaging. IEEE Sens. J. 2015, 15, 3201-3208. [CrossRef]

16. Marchi, G.; Mulloni, V.; Manekiya, M.; Donelli, M.; Lorenzelli, L. A Preliminary Microwave Frequency Characterization of a Nafion-Based Chipless Sensor for Humidity Monitoring. In Proceedings of the 2020 IEEE SENSORS, Yunlin, Taiwan, 29-30 August 2020; pp. 1-4. [CrossRef]

17. Karmakar, N.C.; Amin, E.M.; Saha, J.K., Passive Microwave Design. In Chipless RFID Sensors; Wiley Telecom: Bloomington, CA, USA, 2016; pp. 29-68. [CrossRef]

18. Jilani, M.T.; Rehman, M.Z.; Khan, A.; Chughtai, O.; Abbas, M.; Khan, M. An implementation of IoT-based microwave sensing system for the evaluation of tissues moisture. Microelectron. J. 2018, 88, 117-127. [CrossRef]

19. Lu, Z.; Polizos, G.; Macdonald, D.; Manias, E. State of Water in Perfluorosulfonic Ionomer (Nafion 117) Proton Exchange Membranes. J. Electrochem. Soc. 2008, 155, 2, B163. [CrossRef]

20. Bryant, T.; Weiss, J. Parameters of Microstrip Transmission Lines and of Coupled Pairs of Microstrip Lines. IEEE Trans. Microw. Theory Tech. 1968, 16, 1021-1027. [CrossRef] 